\title{
Nonlinear Alfvén wave model of spicules and coronal heating
}

\author{
Takahiro Kudoh \\ Division of Theoretical Astronomy, National Astronomical Observatory of Japan, Mitaka, \\ Tokyo 181-8588, Japan \\ email:kudoh@th.nao.ac.jp
}

\begin{abstract}
We review the theoretical studies of the Alfvén wave model of spicules and coronal heating, mainly based on the papers by Kudoh \& Shibata (1999), Saito et al. (2001) and Moriyasu et al. (2004) which performed MHD numerical simulations of nonlinear Alfvén waves propagating along a magnetic flux tube in the solar atmosphere. Kudoh \& Shibata (1999) and Saito et al. (2001) found that, if the root mean square of the perturbation is greater than $\sim 1 \mathrm{~km} \mathrm{~s}^{-1}$ in the photosphere, (1) the transition region is lifted up to more than $\sim 5000 \mathrm{~km}$ (i.e., the spicule is produced), (2) the energy flux sufficient for heating the quiet corona $\left(\sim 3.0 \times 10^{5} \mathrm{ergs} \mathrm{s}^{-1} \mathrm{~cm}^{-2}\right)$ is transported into the corona by Alfvén waves. Moriyasu et al. (2004) demonstrated that a hot corona is created in an initially cool loop as a result of the nonlinear Alfvén waves produced near the photosphere. We conclude that the nonlinear Alfvén wave model is the promising model of spicules and coronal heating.
\end{abstract}

Keywords. Sun:chromosphere, Sun:corona, Sun:magnetic fields, Sun:oscillations

\section{Introduction}

Spicules are jets ejected from supergranulation boundaries at supersonic speed $(\sim 30$ $\mathrm{km} \mathrm{s}^{-1}$ ) along magnetic field lines, reaching a height of 5000-7000 km above the photosphere with a lifetime of about 5 minutes (e.g., Beckers 1972; Nishikawa 1988; Suematsu, Wang, \& Zrin 1995; Sterling 2000). As a theoretical model of spicules, Suematsu et al. (1982) and subsequent papers (e.g., Shibata et al. 1982; Hollweg 1982; Sterling, Shibata, \& Mariska 1993) studied gas dynamic shocks that propagate along a magnetic flux tube (i.e., slow-mode MHD shocks) to lift up chromospheric plasmas just below the transition region. On the other hand, Hollweg, Jackson, \& Galloway (1982) and subsequent papers (e.g., Hollweg 1992, Kudoh \& Shibata 1999, Saito, Kudoh, \& Shibata 2001, James, Erdélyi, \& De Pontieu 2003) studied the dynamical effects of nonlinear Alfvén waves propagating in an axisymmetric vertical magnetic flux tube. Their numerical simulation showed that the fast-mode and slow-mode MHD shocks produced in the chromosphere also impel the transition region and underlying chromosphere upward. Recently, from high-resolution observations of dynamic fibrils that are similar objects to the spicules but a little shorter in both length and time scales, De Pontieu et al. (2007) clearly showed the parabolic paths of the dynamic fibrils in the time-distance diagram, which is consistent with shock wave models. They also found the correlation between the maximum velocities and the decelerations of the dynamic fibrils. The correlation is plainly explained by Hansteen et al. (2006) and Heggland, De Pontieu, \& Hansteen (2007) using a shock wave model. Then, shock wave models of spicules are now being promising.

Not only studying spicules, but also Hollweg, Jackson, \& Galloway (1982) argued that the solar corona is heated by the nonlinear Alfvén waves propagating along the flux tubes which produce spicules. Kudoh \& Shibata (1999) carried on more quantitative arguments of their model and concluded that both spicules and the hot quiet corona can 
be caused by Alfvén waves generated at the photosphere if the average amplitude of the transverse perturbation is larger than $\sim 1 \mathrm{~km} \mathrm{~s}^{-1}$. Moriyasu et al. (2004) extended the model including thermal conduction and radiation cooling and demonstrated that the corona is really heated to $10^{6} \mathrm{~K}$ through the dissipation of shock waves excited by the non-linear coupling of Alfvén waves.

The interesting point of the nonlinear Alfvén wave model is that it can explain both spicules (jets in chromosphere) and coronal heating in the same framework. In this article, we will review the nonlinear Alfvén wave model which was first proposed by Hollweg, Jackson, \& Galloway (1982) and followed by Kudoh \& Shibata (1999), Saito et al. (2001), James \& Erdélyi (2002), and Moriyasu et al. (2004).

\section{Formulation of the model}

Hollweg, Jackson, \& Galloway (1982) formulated the 1-dimensional ideal magnetohydrodynamic equations along an axisymmetric flux tube. If we define $s$ as the distance measured along poloidal field line, $r$ is the radius of the flux tube, $z$ is the height of the flux tube, and $\phi$ is the azimuthal angle measured around the rotation axis of the flux tube, the basic equations are as follows: mass conservation,

$$
\frac{\partial \rho}{\partial t}+v_{s} \frac{\partial \rho}{\partial s}=-\rho B_{s} \frac{\partial}{\partial s}\left(\frac{v_{s}}{B_{s}}\right) ;
$$

the $s$ component of the momentum equation,

$$
\frac{\partial v_{s}}{\partial t}+v_{s} \frac{\partial v_{s}}{\partial s}=-\frac{1}{\rho} \frac{\partial p}{\partial s}-g \frac{\partial z}{\partial s}+\frac{v_{\phi}^{2}}{r} \frac{\partial r}{\partial s}-\frac{1}{4 \pi \rho} \frac{B_{\phi}}{r} \frac{\partial}{\partial s}\left(r B_{\phi}\right) ;
$$

the $\phi$ component of the momentum equation,

$$
\frac{\partial\left(r v_{\phi}\right)}{\partial t}+v_{s} \frac{\partial\left(r v_{\phi}\right)}{\partial s}=\frac{B_{s}}{4 \pi \rho} \frac{\partial}{\partial s}\left(r B_{\phi}\right)
$$

the $\phi$ component of the induction equation,

$$
\frac{\partial}{\partial t}\left(\frac{B_{\phi}}{r B_{s}}\right)+\frac{\partial}{\partial s}\left(\frac{B_{\phi}}{r B_{s}} v_{s}-\frac{v_{\phi}}{r}\right)=0 ;
$$

the adiabatic energy equation,

$$
\frac{\partial e}{\partial t}+v_{s} \frac{\partial e}{\partial s}=-(\gamma-1) e B_{s} \frac{\partial}{\partial s}\left(\frac{v_{s}}{B_{s}}\right) ;
$$

and the equation of state, assumed to be that of ideal gas,

$$
e=\frac{1}{\gamma-1} \frac{p}{\rho}
$$

where $\rho$ is the density, $p$ is the pressure, $e$ is the specific internal energy, $v_{s}$ is the velocity along the flux tube, $v_{\phi}$ is the azimuthal component of the velocity, $B_{s}$ is the magnetic field strength along the flux tube, and $B_{\phi}$ is the azimuthal component of the magnetic field, $g$ is the gravitational acceleration, and $\gamma$ is the specific heat ratio.

In the 1-dimensional approximation, we do not consider the force balance perpendicular to the flux tube. In this formulation, $B_{s}$ is given as a function of coordinates. Normally, $B_{s} \propto r^{-2}$ is taken for simplicity. The relation among $s, r$, and $z$ are also given with the shape of the flux tube. Hollweg, Jackson, \& Galloway (1982), Kudoh \& Shibata (1999), and Saito, Kudoh, \& Shibata (2001) assumed open flux tubes like Fig.1 in Kudoh \& 
Shibata (1999). Moriyasu et al. (2004) assumed a closed flux tube in Fig.1 of their paper. In both cases, they assumed small cross sections of flux tubes at the photosphere and large cross sections in the corona, assuming that $B_{s}$ in the corona is smaller than that at the photosphere.

The formulation presented above describes the torsional motion of the flux tube. The differences between the torsional and the normal transverse motions (e.g., kink mode of the flux tube) are the centrifugal force in the $s$ component of the momentum equation and $r$ or $1 / r$ factors appeared before $B_{\phi}$ and $v_{\phi}$ in the equations $(2.2)-(2.4)$. When the flux tube has constant cross section (i.e., $\partial r / \partial s=0$ ), equations show that these are the same as those of the normal transverse motion.

Numerical simulation is a powerful tool to study spicules, because spicules are considered to be nonlinear phenomena. Then, spicules has been studied by solving those one-dimensional equations numerically. Although the above equations are for studying the Alfvén wave models, the same equations can be used for gasdynamic shock models if we only consider the motion along the flux tube with $B_{\phi}=v_{\phi}=0$. The gasdynamic shock model assumed the vertical oscillation at the photosphere, while the Alfvén wave model assumed the transverse oscillation at the photosphere.

\section{Spicule formation and coronal heating}

Suematsu et al. (1982) did a numerical simulation using the 1-dimensional equations and input pressure pulse at the footpoint of flux tubes (at the photosphere). This corresponds to inputting an artificial vertical force into the $s$ component of the momentum equation (2.2). They found that the gasdynamic shocks (slow-mode MHD shocks) which are developed in the gravitationally stratified atmosphere lifted up the chromosphere. The input pressure pulse corresponds to the vertical oscillation on the photosphere. In this case, no Alfvén waves appeared in the flux tube $\left(B_{\phi}=v_{\phi}=0\right)$.

Instead of inputting a pulse into the vertical momentum equations, Hollweg, Jackson, $\&$ Galloway (1982) input a pulse into the $\phi$ component of the momentum equation (2.3) as an artificial torsional force. This torsional force produces an Alfvén wave into the flux tube. If the artificial force is very weak, it just propagates into the flux tube as a linear Alfvén wave. However, when the amplitude is large (or the frequency is small), they found that the fast-mode MHD shock was formed in the chromosphere by the nonlinear effect of the Alfvén wave (i.e., the magnetic pressure term in the equation (2.3) which is negligible when $B_{\phi}$ is small) and the chromosphere was lifted up by the shock. Not only lifting up the chromosphere, but also the part of the fast shock wave propagates into the corona. They found that the energy flux of the fast shock waves in the corona was larger than the energy flux which is expected for heating of the quiet corona. These nonlinear effects happened when the amplitude of the perturbation is $1.78 \mathrm{~km} \mathrm{~s}^{-1}$ and the period is 90.26 s at the photosphere, for example. Hollweg (1992) also argued the formation of the slow-mode MHD shock in the chromosphere behind the transverse pulse. The slowmode shock formed by the nonlinear effect of the Alfvén waves also lifts up the transition region.

Kudoh \& Shibata (1999) did similar MHD simulations to that of Hollweg, Jackson, \& Galloway (1982) and carried on more quantitative arguments of the Alfvén wave model. Instead of inputting the pulse, Kudoh \& Shibata (1999) input a random continuous transverse force into the flux tube. The frequency of the random motion is roughly between $10^{-3} \mathrm{~Hz}$ and $10^{-1} \mathrm{~Hz}$. If the root mean square of the velocity amplitude is larger than about $1 \mathrm{~km} \mathrm{~s}^{-1}$ at the photosphere, they found that: (1) The transition region is lifted up to more than $\sim 5000 \mathrm{~km}$ (i.e., the spicule is produced) by the 
slow- and fast-mode MHD waves (shocks) produced by the nonlinear effect of torsional Alfvén waves. (2) The energy flux sufficient for the heating of the quiet corona (i.e., $\sim 3.0 \times 10^{5} \mathrm{erg} \mathrm{s}^{-1} \mathrm{~cm}^{-2}$ ) is transported into the corona as Alfvén waves. (3) Nonthermal broadening of emission lines in the corona is about $20 \mathrm{~km} \mathrm{~s}^{-1}$ and its dependence on the temperature is consistent with the observations. The result of (2) and (3) are related each other. Both of them are caused by the Alfvén waves propagation in the corona. It is interesting that the velocity perturbation of the $1 \mathrm{~km} \mathrm{~s}^{-1}$ is the typical velocity at the photosphere.

Saito, Kudoh \& Shibata (2001) studied both cases of inputting the vertical force and inputting the transverse force into the footpoint of the flux tube, and compared these two models. They found that the taller spicules are produced by the vertical force than the transverse force if the input energy is the same. They also found that the slow-mode MHD waves which are formed by the nonlinear effect of the Alfvén waves lift up the transition region more effectively than the fast-mode MHD waves. It means that the slow-mode MHD wave play a more fundamental role in the generation of spicules even in the case of inputting the transverse force. However, in the case of inputting vertical force, the energy propagating into the corona is not sufficient for the heating of corona even if the input energy is very large, because the slow-mode waves easily dissipate in the chromosphere. They concluded that; (1) The spicules are mainly produced by the slow-mode MHD waves, even when the transverse force is input. (2) The slow-mode MHD waves alone are not enough for the heating of corona.

James \& Erdélyi (2002) did similar numerical simulations with inputting the transverse force into the footpoint of the flux tube. They studied the effect of ion-neutral damping in spicule formation, which was proposed by Haerendel (1992). James \& Erdélyi (2002) and James, Erdélyi, \& De Pontieu (2003) found that the damping was not efficient for the spicule formation. The spicule was mainly produced by slow-mode MHD shocks generated from the nonlinear effect of the Alfvén waves, in the same way as Saito, Kudoh, \& Shibata (2001) concluded. However, they found that the dissipation due to the ionneutral damping significantly contributed to the heating of chromospheric plasma.

Moriyasu et al. (2004) extended the Alfvén wave models to the closed magnetic loop. He studied the problem of coronal heating, including thermal conduction and radiation cooling. He clearly showed that the corona is heated to $10^{6} \mathrm{~K}$ by fast- and slow-mode MHD shocks generated by nonlinear Alfvén waves. Since the heating is episodic, the time evolution of the pseudo-intensities obtained from the simulation shows that they are very similar to those of the X-ray and EUV intensities observed with SXT and TRACE (Fig.3 in Moriyasu et al. (2004)). The histogram of occurrence frequency of these theoretical nanoflares is also similar to that observed in the solar corona (Fig.4 in Moriyasu et al. (2004)). These suggests that the nanoflare-like events would be due to the MHD shocks originally generated by the Alfvén waves. This will be an alternative model of nanoflares by magnetic reconnection (Parker 1988). Moriyasu et al. (2004) also did a parameter survey of this model and found that the steady corona whose temperature is $\sim 10^{6} \mathrm{~K}$ is achieved if the velocity amplitude of the transverse perturbation is larger than about $1 \mathrm{~km} \mathrm{~s}^{-1}$ (see also Moriyasu \& Shibata (2004)). This result is consistent with that of Kudoh \& Shibata (1999).

\section{Conclusions and Discussion}

We reviewed the theoretical studies of the Alfvén wave model of spicules and coronal heating. In this model, the Alfvén waves are assumed to be generated by the transverse perturbation at the footpoint of the flux tube (at the photosphere). When they propagate 
in the chromosphere, the nonlinear effects of the Alfvén waves form slow- and fast-mode MHD shocks which lift up the chromosphere (i.e., spicule formation) and heat the corona. The interesting point of the Alfvén wave model is that it is the model both of spicules and the coronal heating in the same framework. If there is a transverse perturbation which is larger than about $1 \mathrm{~km} \mathrm{~s}^{-1}$, the chromosphere is lifted to about $5000 \mathrm{~km}$ and the corona is heated to $10^{6} \mathrm{~K}$.

Concerning about the spicules, the Alfvén wave model looks the alternative model to the gasdynamic shock model. In the gasdynamic model, the acoustic waves are assumed to be generated by the field-aligned oscillation at the footpoints, and the chromosphere is lifted up by the slow-mode MHD shocks generated in the gravitationally stratified atmosphere. However, even in the Alfvén wave model, the slow-mode MHD waves play a more fundamental role in the generation of spicules (Saito, Kudoh, \& Shibata 2001). In this sense, the difference is how to make slow-mode MHD waves in the flux tube. In the Alfvén wave model, the slow-mode MHD waves are formed through the nonlinear effect of the Alfvén waves generated by the transverse oscillation at the photosphere. In the gasdynamic shock model, it is directly formed from the field-aligned oscillation at the photosphere. Photospheric velocities that move the magnetic flux tube will be caused by convective granulation (Sterling \& Mariska 1990), magnetic reconnection in the lower chromosphere (Takeuchi \& Shibata 2001), and solar global acoustic oscillations (Suematsu 1990, De Pontieu, Erdélyi, \& James 2004, De Pontieu, \& Erdélyi 2006 ). In the real solar atmosphere, both the vertical and transverse oscillation would be important to consider the formation of spicules.

Concerning about coronal heating, the Alfvén wave model is the alternative model to the magnetic reconnection model (Parker 1988). Parker proposed nanoflare heating in which many small and localized bursts of energy is released through magnetic reconnection in loops as a consequence of random continuous motion of the footpoints of the field in the photospheric convection. In Moriyasu et al. (2004)'s model, the small and localized bursts of energy is released though the shock dissipation as a consequence of the nonlinear effects of Alfvén waves caused by random continuous motion of the footpoints of the field. Antolin et al. (2007) recently compared these two models and found different histograms of occurrence frequency of these theoretical nanoflares. These two models may be compared with observations more quantitatively by using recent new diagnostic tool proposed by Taroyan et al. (2007).

The formulation proposed by Hollweg, Jackson, \& Galloway (1982) is the simple way to study Alfvén waves in a low $\beta$ flux tube whose cross section changes with height in the 1-dimensional approximation. It describes the torsional motion of the flux tube. Some people do not think it is likely that the torsional motions effectively perturb flux tubes at the photosphere. However, if the flux tube has constant cross section (i.e., $\partial r / \partial s=0)$, equations are the same as those of the normal transverse motion (e.g., kink mode of the flux tube). The differences between the equations of the torsional motions and those of the transverse motions are the centrifugal force in the $s$ component of the momentum equation and $r$ or $1 / r$ factors appeared before $B_{\phi}$ and $v_{\phi}$ in the equations (2.2) - (2.4). Then, we believe that the results obtained from Hollweg's formulation would be approximately applicable to the flux tubes perturbed with kink mode oscillations unless the centrifugal force is very important. This should be confirmed in 2-dimensional or 3-dimensional MHD simulations in the near future.

The idea of the Alfvén wave model is recently applied to some astrophysical objects, such as the solar wind (Suzuki \& Inutsuka 2005), red giants winds (Suzuki 2007), molecular cloud turbulence (Kudoh \& Basu 2003; 2006), and the heating of galaxy clusters 
(Fujita et al. 2007). It is also important to consider the similarity of the physics in these astrophysical objects and get feedback them to the solar phenomena, and vice versa.

\section{Acknowledgements}

The author was supported by the Foundation for Promotion of Astronomy in Japan and IAU Grant to attend the symposium.

\section{References}

Antolin, P., Shibata, K., Kudoh, T., Shiota, D., \& Brooks D. 2008, in R. Erdélyi and C.A. Mendoza-Briceño (eds.) Waves $\&$ Oscillations in the Solar Atmosphere: Heating and Magneto-seismology, IAU Symposyum 247, 281

Beckers, J.M. 1972, ARAA 10, 73

De Pontieu, B., Erdélyi, R., \& James, S.P. 2004, Nature 430, 536

De Pontieu, B., \& Erdélyi, R. 2006, Phil. Trans. R. Soc. A 364, 383

De Pontieu, B., Hansteen, V.H., Rouppe van der Voort, L., van Noort, M., \& Carlsson, M. 2007, ApJ 655, 624

Fujita, Y., Suzuki, T.K., Kudoh, T., \& Yokoyama, T. 2007, ApJ (Letters) 659, L1

Haerendel, G. 1992, Nature 360, 241

Hansteen, V.H., De Pontieu, B., Rouppe van der Voort, L., van Noort, M., \& Carlsson, M. 2006, ApJ (Letters) 647, L73

Heggland, L., De Pontieu, B., \& Hansteen, V.H. 2007, ApJ 666, 1277

Hollweg, J.V. 1982, ApJ 257, 345

Hollweg, J.V., Jackson, S., \& Galloway, D. 1982, Solar Phys. 75, 35

Hollweg, J.V. 1992, ApJ 389, 731

James, S.P., \& Erdélyi, R. 2002, A\&A (Letters) 393, L11

James, S.P., Erdélyi, R., \& De Pontieu, B. 2003, A\&A 406, 715

Kudoh, T., \& Shibata, K. 1999, ApJ 514, 493

Kudoh, T., \& Basu, S. 2003, ApJ 595, 842

Kudoh, T., \& Basu, S. 2006, ApJ 642, 270

Moriyasu, S., Kudoh, T., Yokoyama, T., \& Shibata, K. 2004, ApJ (Letters) 601, L107

Moriyasu, S., \& Shibata K. 2004, in: R.W. Walsh, J. Ireland, D. Danesy \& B. Fleck (eds.), Proceedings of the SOHO 15 Workshop - Coronal Heating, (Paris: ESA), ESASP-575, p. 80

Nishikawa, T. 1988, PASJ 40, 613

Parker, E.N. 1988, ApJ 330, 474

Saito, T., Kudoh, T., \& Shibata, K. 2001, ApJ 554, 1151

Shibata, K., Nishikawa, T., Kitai, R., \& Suematsu, Y. 1982, Solar Phys. 77, 121

Sterling, A.C., \& Mariska, J.T. 1990, ApJ 349, 647

Sterling, A.C. 2000, Solar Phys. 196, 79

Sterling, A.C., Shibata, K., \& Mariska, J.T. 1993, ApJ 407, 778

Suematsu, Y., Shibata, K., Nishikawa, T., \& Kitai, R. 1982, Solar Phys. 75, 99

Suematsu, Y. 1990, in: Y. Osaki \& H. Shibahashi (eds.), Progress of Seismology of the Sun and Stars, (Berlin: Springer), p. 211

Suematsu, Y., Wang, H., \& Zrin, H. 1995, ApJ 450, 411

Suzuki, T.K., \& Inutsuka, S. 2005, ApJ (Letters) 632, L49

Suzuki, T.K. 2007, ApJ 659, 1592

Takeuchi, A, \& Shibata, K. 2001, ApJ (Letters) 546, L73

Taroyan, Y., Erdélyi, R., Doyle, J.G., \& Bradshaw, S.J. 2007, A\&A 462, 331 